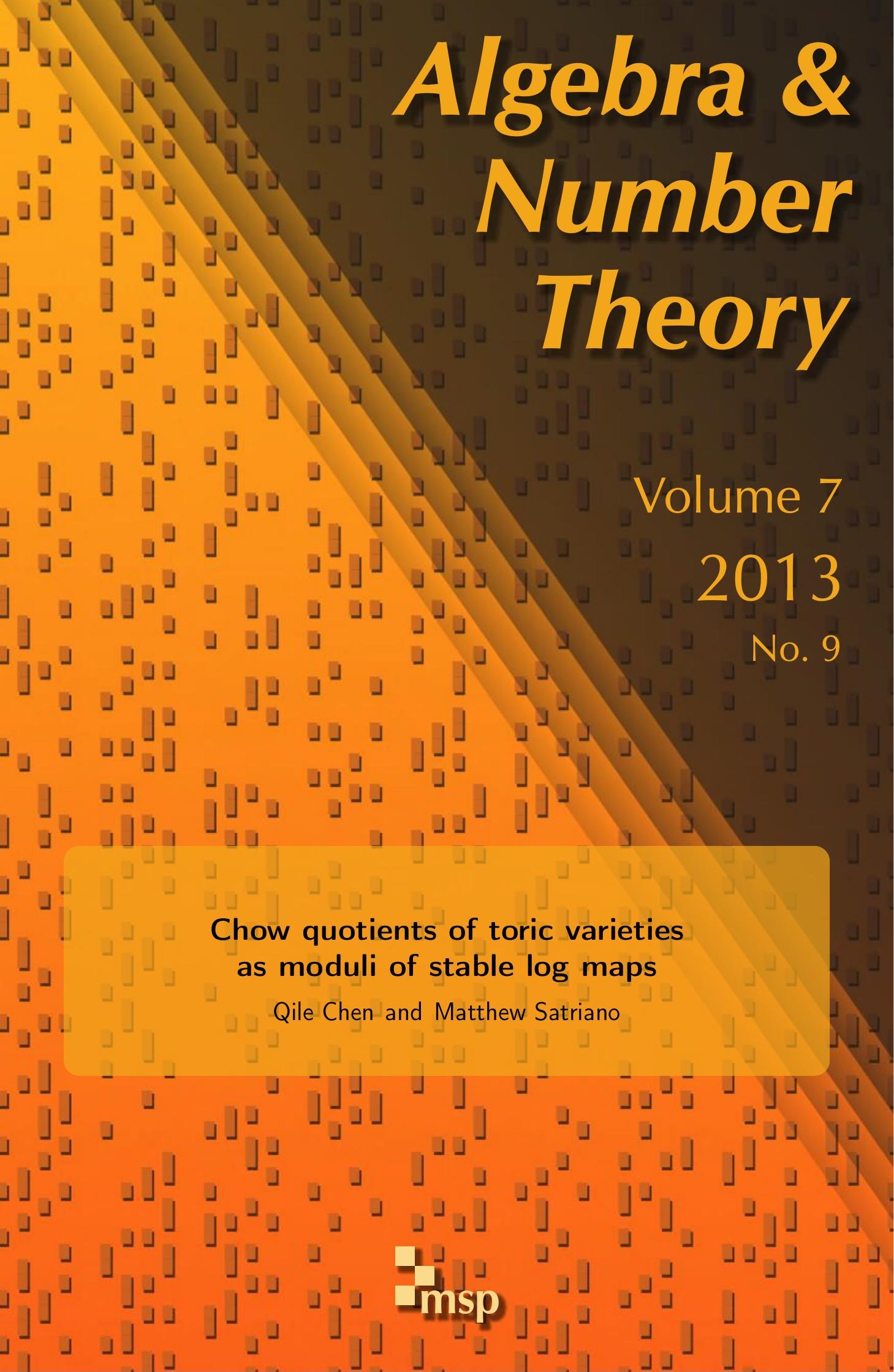




\title{
Chow quotients of toric varieties as moduli of stable log maps
}

\author{
Qile Chen and Matthew Satriano
}

Let $X$ be a projective normal toric variety and $T_{0}$ a rank-1 subtorus of the defining torus $T$ of $X$. We show that the normalization of the Chow quotient $X / / T_{0}$, in the sense of Kapranov, Sturmfels, and Zelevinsky, coarsely represents the moduli space of stable $\log$ maps to $X$ with discrete data given by $T_{0} \subset X$. We also obtain similar results when $T_{0} \rightarrow T$ is a homomorphism that is not necessarily an embedding.

1. Introduction

2. Log smoothness and irreducibility

3. Tropical curves associated to stable log maps

4. The Chow quotient as the coarse moduli space

Appendix: Toric varieties have generalized Deligne-Faltings log structures

Acknowledgments

References

2328

\section{Introduction}

Throughout, we work over an algebraically closed field $k$ of characteristic 0 .

Chow quotients of toric varieties were introduced by Kapranov, Sturmfels, and Zelevinsky in [Kapranov et al. 1991]. Given a projective normal toric variety $X$ and a subtorus $T_{0}$ of the defining torus $T$, the Chow quotient $X / / T_{0}$ has the property that its normalization is the smallest toric variety that maps onto all GIT quotients of $X$ by $T_{0}$. We show in this paper that when $T_{0}$ has rank 1 , the normalization of $X / / T_{0}$ can be reinterpreted as the coarse moduli space of the stack of stable log maps introduced in [Chen 2011; Abramovich and Chen 2011] and independently in [Gross and Siebert 2013]. We also obtain similar results by replacing $T_{0} \subset T$ with a homomorphism $T_{0} \rightarrow T$ and the Chow quotient in the sense of [Kapranov et al. 1991] with that of [Kollár 1996].

MSC2010: primary 14H10; secondary 14N35.

Keywords: toric, Kontsevich, stable log map, Chow quotient. 
Let $X$ be a normal toric variety of dimension $n$ with defining torus $T$. Denoting by $N \cong \mathbb{Z}^{n}$ the cocharacter lattice of $T$, we see that every point $v \in N$ corresponds to a morphism of multiplicative groups

$$
\iota_{v}: T_{0}:=\mathbb{G}_{m} \rightarrow T \text {. }
$$

It is convenient to view this map as the action of $T_{0}$ on the identity element $1 \in T$. Let $v=k \omega$ for some positive integer $k$ and primitive lattice point $\omega \in N$. Note that $\iota$ is an embedding if and only if $k=1$.

We begin by introducing the Chow quotient $X / / T_{0}$. For every point $x \in T$, the closure $Z_{x}:=\overline{T_{0} x}$ of the orbit of $x$ under $T_{0}$ with the reduced scheme structure is a subvariety of $X$. Thus, we obtain a Chow cycle $k \cdot\left[Z_{x}\right]$. For $x \in T$, the orbit closures $Z_{x}$ have the same dimension and homology class. Denoting by $T^{\prime}:=\left[T / T_{0}\right]$ the stack quotient, we therefore obtain a morphism from $T^{\prime}$ to the Chow variety $C(X)$ of algebraic cycles of the given dimension and homology class. For the definition and construction of the Chow variety $C(X)$, we refer to [Kollár 1996, Chapter I]. Since the Chow variety is not actually a moduli space for cycles as above, one may initially be worried that we only obtain a map on the level of closed points. However, we will later see that there is a family of stable maps over $T^{\prime}$ whose image is precisely the Chow cycle we obtained here; it then follows from [Kollár 1996, Chapter I, 3.17 and 3.21] that there is a natural map $T^{\prime} \rightarrow C(X)$. We define the Chow quotient $X / / T_{0}$ to be the closure of the image $T^{\prime}$ in $C(X)$ with the reduced scheme structure.

Note that when $k=1, T^{\prime}$ is a variety and $X / / T_{0}$ is the Chow quotient introduced by Kapranov et al. [1991]. In this case, it is a toric variety and the fan of its normalization is given explicitly in [Kapranov et al. 1991, §1].

As mentioned above, the goal of this paper is to relate $X / / T_{0}$ to moduli spaces of stable $\log$ maps. Notice that by compactifying $\iota$, we obtain a stable map $f_{1}: \mathbb{P}^{1} \rightarrow X$, where $\mathbb{P}^{1}$ is marked at the points $\{0, \infty\}=\mathbb{P}^{1} \backslash T_{0}$. By viewing $X$ as a log scheme with its canonical $\log$ structure $M_{X}$ given by the boundary $X \backslash T$ and $\mathbb{P}^{1}$ as a log curve with $\log$ structure $\mathcal{M}_{\mathbb{P}^{1}}$ given by the two markings $\{0, \infty\}$, we obtain a stable $\log$ map

$$
f_{1}:\left(\mathbb{P}^{1}, M_{\mathbb{P}^{1}}\right) \rightarrow\left(X, \mu_{X}\right) .
$$

Let $\beta_{0}$ be the curve class of the stable map $f_{1}$, and let $c_{0}$ and $c_{\infty}$ be the contact orders of 0 and $\infty$ with respect to the toric boundary $X \backslash T$. Roughly speaking, $c_{0}$ and $c_{\infty}$ are functions that assign to the marked points their orders of tangency with the components of $X \backslash T$ (see [Abramovich et al. 2011] for more details). In the toric case, the contact orders can be explained as the slopes and weights of the unbounded edges of tropical curves associated to stable log maps; see Section 3.3. Let $\mathscr{K}_{\Gamma_{0}}(X)$ be the stack parametrizing stable log maps from rational curves with 
two marked points to $X$ such that the curve class is $\beta_{0}$ and the marked points have contact orders given by $c_{0}$ and $c_{\infty}$; here the notation

$$
\Gamma_{0}:=\left(0, \beta_{0}, 2,\left\{c_{0}, c_{\infty}\right\}\right)
$$

keeps track of the discrete data consisting of genus, curve class, number of marked points, and their tangency conditions. Our main result is:

Theorem 1.1. The normalization of $X / / T_{0}$ is the coarse moduli space of $\mathscr{K}_{\Gamma_{0}}(X)$.

Remark 1.2. In particular, we see that $\mathscr{K}_{\Gamma_{0}}(X)$ is irreducible.

Remark 1.3. In Proposition 2.3, we prove that for any $\Gamma=\left(0, \beta, 2,\left\{c_{0}, c_{\infty}\right\}\right)$, either the stack $\mathscr{K}_{\Gamma}(X)$ is empty or $\Gamma=\Gamma_{0}$ for some $\Gamma_{0}$ as in (1-2). Thus, our discussion covers all two-pointed stable log maps to toric varieties.

In the process of proving Theorem 1.1, we obtain an alternative description of $\mathscr{K}_{\Gamma_{0}}(X)$ that is more akin to the construction of the Chow quotient. As we saw above, $X / / T_{0}$ is defined as the closure of $T^{\prime}$ in the Chow variety $C(X)$. Replacing $C(X)$ by other moduli spaces, we obtain alternate spaces analogous to $X / / T_{0}$. For each point $x \in T$, letting $T_{0}$ act on $x$ via the group morphism $\iota$ and taking the closure, we obtain a stable log map

$$
f_{x}:\left(\mathbb{P}^{1}, \mu_{\mathbb{P} 1}\right) \rightarrow\left(X, \mu_{X}\right)
$$

again with curve class $\beta_{0}$ and contact orders $c_{0}$ and $c_{\infty}$. Note that for any point $x^{\prime} \in \overline{T_{0} x}$, the two stable $\log$ maps $f_{x}$ and $f_{x^{\prime}}$ are canonically isomorphic. We thus obtain a family of stable log maps over the stack quotient $T^{\prime}$. It is important to notice that the $\log$ structure on $T^{\prime}$ is trivial (and is denoted by $0^{*}$ ). The stack $\mathscr{K}_{\Gamma_{0}}(X)$ comes equipped with a $\log$ structure, and the above discussion defines a morphism of log stacks

$$
\left(T^{\prime}, \mathcal{O}_{T^{\prime}}^{*}\right) \rightarrow\left(\mathscr{K}_{\Gamma_{0}}(X), M_{\mathscr{K}_{\Gamma_{0}}(X)}\right) .
$$

Forgetting the log structures, we obtain an immersion

$$
T^{\prime} \rightarrow \mathfrak{M}_{0,2}\left(X, \beta_{0}\right),
$$

where $\mathfrak{M}_{0,2}\left(X, \beta_{0}\right)$ denotes the Kontsevich space of stable maps to $X$ with genus 0 , curve class $\beta_{0}$, and two marked points. In analogy with the construction of the Chow variety, we let $\mathfrak{M}$ denote the closure of $T^{\prime}$ in $\mathfrak{M}_{0,2}\left(X, \beta_{0}\right)$. Then we have:

Theorem 1.4. $\mathcal{K}_{\Gamma_{0}}(X)$ is the normalization of $\mathfrak{M}$.

Remark 1.5. There is an analogous picture if one assumes that $X$ is an affine normal toric variety and replaces $\mathfrak{M}_{0,2}\left(X, \beta_{0}\right)$ above by the toric Hilbert scheme, as defined in [Peeva and Stillman 2002]. That is, for all $x \in T$, the $Z_{x}$ are $T^{\prime}$-invariant closed subschemes of $X$ that have the same discrete invariants. We therefore 
obtain an immersion from $T^{\prime}$ to an appropriate toric Hilbert scheme. The closure of $T^{\prime}$ in this toric Hilbert scheme is called the main component. Olsson [2008, Theorem 1.7] shows that the normalization of the main component has a natural moduli interpretation in terms of log geometry. Theorem 1.4 above can therefore be viewed as an analogue of Olsson's theorem, replacing his use of the toric Hilbert scheme by the Kontsevich space. That is, we show that the normalization of $\mathfrak{M}$ carries a moduli interpretation in terms of stable log maps.

Recall that given any collection of discrete data $\Gamma=\left(g, \beta, n,\left\{c_{i}\right\}_{i=1}^{n}\right)$, it is shown in [Chen 2011; Abramovich and Chen 2011; Gross and Siebert 2013] that there is a proper Deligne-Mumford stack $\mathscr{K}_{\Gamma}(X)$ that parametrizes stable log maps to $X$ from genus- $g$ curves with $n$ marked points having curve class $\beta$ and contact orders given by the $c_{i}{ }^{1}$ We show in Proposition 2.1 that if $g=0$, then $\mathscr{K}_{\Gamma}(X)$ is $\log$ smooth and in particular normal. This is a key ingredient in the proof of Theorem 1.4, which we give in Section 2. In Section 3, following [Nishinou and Siebert 2006; Gross and Siebert 2013], we explain the relationship between tropical curves and stable $\log$ maps to toric varieties. While the use of tropical curves is not strictly necessary for this paper, they serve as a convenient tool to study the boundary of $\mathscr{K}_{\Gamma}(X)$. Theorem 1.1 is then proved in Section 4.

Remark 1.6. One of the purposes of the theory of stable log maps is to define and compute Gromov-Witten invariants with tangency conditions. The authors plan to calculate the Gromov-Witten invariants in the case of this paper once the forthcoming paper [Abramovich et al. $\geq 2013$ ] is ready to use; this latter paper will carefully treat the virtual cycle of the space of stable log maps as well as a version of the degeneration formula of Gromov-Witten invariants.

Prerequisites. We assume the reader is familiar with logarithmic geometry in the sense of Fontaine, Illusie, and Kato (see for example [Kato 1989] or [Ogus 2006]).

\section{Log smoothness and irreducibility}

Throughout this section, $X$ is a projective normal toric variety of dimension $d$ and $\Gamma$ is an arbitrary choice of discrete data $\left(0, \beta, n,\left\{c_{i}\right\}\right)$. Let $T$ be the defining torus of $X$ and $M$ be the character lattice of $T$.

Proposition 2.1. $\left(\mathscr{K}_{\Gamma}(X), \mathcal{M}_{\mathscr{K}_{\Gamma}(X)}\right)$ is log smooth over $\left(k, \mathcal{O}_{k}^{*}\right) \cdot$ Also, $\operatorname{dim} \mathscr{K}_{\Gamma}(X)=$ $\operatorname{dim} X+n-3$.

\footnotetext{
${ }^{1}$ Strictly speaking, [Chen 2011; Abramovich and Chen 2011] only consider log schemes that are generalized Deligne-Faltings (see Definition A.1), so to apply their theory, one must first show that the natural $\log$ structure on $X$ satisfies this hypothesis. This is done in Proposition A.4, which we relegate to the Appendix since the theory developed in [Gross and Siebert 2013] is already known to apply to toric varieties.
} 
Proof. The universal curve on $\mathscr{K}_{\Gamma}(X)$ induces a morphism of log stacks

$$
\pi:\left(\mathscr{K}_{\Gamma}(X), \mathcal{M}_{\mathscr{K}_{\Gamma}(X)}\right) \rightarrow\left(\mathfrak{M}_{0, n}, \mathcal{M}_{\mathfrak{M}_{0, n}}\right),
$$

where $\left(\mathfrak{M}_{g, n}, M_{\mathfrak{M}_{g, n}}\right)$ denotes the log stack of $(g, n)$-prestable curves; see [Kato 2000] and [Olsson 2007, Theorem 1.10] for the definition and construction of this log stack. Since $\left(\mathfrak{M}_{g, n}, M_{\mathfrak{M}_{g, n}}\right)$ is $\log$ smooth over $\left(k, O_{k}^{*}\right)$, it suffices to show that $\pi$ is log smooth. By [Olsson 2003, Theorem 4.6], this is equivalent to showing that the induced morphism

$$
\pi^{\prime}: \mathscr{K}_{\Gamma}(X) \rightarrow \mathscr{L}_{\left(\mathfrak{M}_{0, n}, M_{\mathfrak{M}_{0, n}}\right)}
$$

of stacks is smooth, where $\log _{\left(S, M_{S}\right)}$ is the stack of $\log$ morphisms to a log scheme $\left(S, M_{S}\right)$ as defined in the introduction of [loc. cit.].

Let $i: \operatorname{Spec} A \rightarrow \operatorname{Spec} A^{\prime}$ be a square zero thickening of Artin local rings, and let

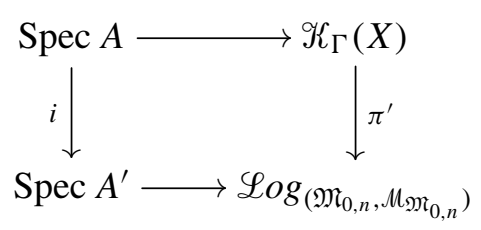

be a commutative diagram. We may view this as a commutative diagram of log stacks by endowing the Artin local rings with the log structure pulled back from $\mathscr{L}_{\left(\mathfrak{M}_{0, n}, M_{\mathfrak{M}_{0, n}}\right)}$. Hence, the two vertical arrows are strict. Denote the induced log structures on Spec $A$ and Spec $A^{\prime}$ by $M_{A}$ and $M_{A^{\prime}}$, respectively. We therefore have a $\log$ smooth curve $h^{\prime}$, a cartesian diagram

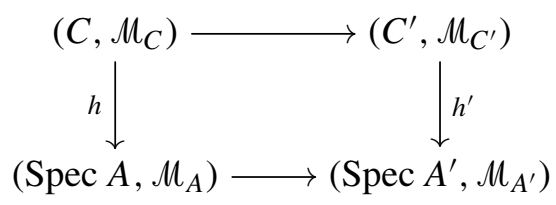

and a minimal stable log map $f:\left(C, \mu_{C}\right) \rightarrow\left(X, \mu_{X}\right)$, which we must show deforms to a minimal stable $\log$ map $f^{\prime}:\left(C^{\prime}, M_{C^{\prime}}\right) \rightarrow\left(X, M_{X}\right)$. Since the minimality condition is open by [Chen 2011, Proposition 3.5.2], it suffices to show that $f$ deforms as a morphism of log schemes.

By standard arguments in deformation theory, it is enough to consider the case where the kernel $\mathscr{I}$ of $A^{\prime} \rightarrow A$ is principal and killed by the maximal ideal $\mathfrak{m}$ of $A^{\prime}$. Then the obstruction to deforming $f$ to a morphism of log schemes lies in

$$
\operatorname{Ext}^{1}\left(f_{0}^{*} \Omega_{\left(X, M_{X}\right) / k}^{1}, O_{C_{0}}\right) \otimes_{k} \mathscr{T},
$$

where $f_{0}$ denotes the reduction of $f \bmod \mathfrak{m}$ and $C_{0}$ denotes the fiber of $C$ over $A^{\prime} / \mathfrak{m}=k$. By [Kato 1996, Example 5.6], $\Omega_{\left(X, M_{X}\right) / k}^{1} \simeq 0_{X} \otimes_{\mathbb{Z}} M$. Therefore, 


$$
\operatorname{Ext}^{1}\left(f_{0}^{*} \Omega_{\left(X, M_{X}\right) / k}^{1}, \mathscr{O}_{C_{0}}\right)=H^{1}\left(\mathcal{O}_{C_{0}}^{d}\right)=0,
$$

where the last equality holds because $C_{0}$ is a curve of arithmetic genus 0 . This shows that $\left(\mathscr{K}_{\Gamma}(X), M_{\mathscr{K}_{\Gamma}(X)}\right)$ is log smooth.

To prove the claim about the dimension of $\mathscr{K}_{\Gamma}(X)$, note that

$$
\operatorname{dim} \operatorname{Ext}^{0}\left(f_{0}^{*} \Omega_{\left(X, \mu_{X}\right) / k}^{1}, O_{C_{0}}\right)=\operatorname{dim} H^{0}\left(O_{C_{0}}^{d}\right)=d,
$$

and so $\pi$ has relative dimension $d$. Since $\operatorname{dim} \mathfrak{M}_{0, n}=n-3$, we see $\operatorname{dim} \mathscr{K}_{\Gamma}(X)=$ $d+n-3$.

Let $\mathscr{K}_{\Gamma}^{\circ}(X)$ denote the nondegeneracy locus, that is, the locus of $\mathscr{K}_{\Gamma}(X)$ where the $\log$ structure $\mathcal{M}_{\mathscr{Y}_{\Gamma}(X)}$ is trivial. By Proposition 2.1 and [Nizioł 2006, Proposition 2.6], $\mathscr{K}_{\Gamma}^{\circ}(X)$ is an open dense subset of $\mathscr{K}_{\Gamma}(X)$. Consider the Kontsevich moduli space of stable maps $\mathfrak{M}_{0, n}(X, \beta)$. The forgetful map

$$
\Phi: \mathscr{K}_{\Gamma}(X) \rightarrow \mathfrak{M}_{0, n}(X, \beta)
$$

sending a stable log map to its underlying stable map induces a locally closed immersion

$$
\mathcal{K}_{\Gamma}^{\circ}(X) \rightarrow \mathfrak{M}_{0, n}(X, \beta) .
$$

Since the forgetful map does not change the underlying markings or the underlying maps, no stabilization of the underlying curve is needed here. Let $\mathfrak{M}_{\Gamma}(X)$ be the closure of $\mathscr{K}_{\Gamma}^{\circ}(X)$ in $\mathfrak{M}_{0, n}(X, \beta)$. Then $\Phi$ factors through a morphism

$$
\phi: \mathscr{K}_{\Gamma}(X) \rightarrow \mathfrak{M}_{\Gamma}(X) .
$$

\section{Lemma 2.2. $\phi$ is the normalization map.}

Proof. By [Abramovich and Chen 2011, Corollary 3.10] and Proposition A.4, the morphism $\Phi$ is representable and finite and so is $\phi$. Since $\left(\mathscr{K}_{\Gamma}(X), M_{\mathscr{K}_{\Gamma}(X)}\right)$ is fs and $\log$ smooth over $\left(k, \mathscr{O}_{k}^{*}\right)$ by Proposition 2.1, it follows that $\mathscr{K}_{\Gamma}(X)$ is normal. Since $\phi$ is an isomorphism over $\mathcal{K}_{\Gamma}^{\circ}(X)$, it is birational, and so by Zariski's main theorem, $\phi$ is the normalization map.

Now we consider the case $\Gamma=\left(0, \beta, 2,\left\{c_{0}, c_{\infty}\right\}\right)$, where $\beta$ is an arbitrary curve class, and $c_{0}$ and $c_{\infty}$ are two arbitrary contact orders along the two different markings. Note that both $c_{0}$ and $c_{\infty}$ are nontrivial. Otherwise, there is a curve in toric variety intersect the boundary at only one point, which is impossible. Then we have the following result:

Proposition 2.3. (1) If $\mathscr{K}_{\Gamma}(X) \neq \varnothing$, then $\Gamma=\Gamma_{0}$ for some $\Gamma_{0}$ as in (1-2), obtained from a group morphism (1-1).

(2) $\mathscr{K}_{\Gamma_{0}}(X)$ is irreducible. 
Proof. Assume that $\mathscr{K}_{\Gamma}(X) \neq \varnothing$; hence, $\mathscr{K}_{\Gamma}^{\circ}(X) \neq \varnothing$ by Proposition 2.1. Let $s \in \mathscr{K}_{\Gamma}^{\circ}(X)(k)$ and $f: \mathbb{P}^{1} \rightarrow X$ be the stable log map corresponding to $s$. Note that the $\log$ structure of the boundary of $X$ is everywhere nontrivial. Since the log structure is trivial at $s$, the image of $f$ necessarily meets $T$. After acting on $f$ by some element of $T$, we may assume that $f$ sends $1 \in \mathbb{P}^{1}$ to $1 \in T \subset X$. Choose a maximal cone $\sigma$ in the fan of $X$ such that the associated affine open toric variety $U \subset X$ contains $f(0)$. Restricting $f$ to $U$, we obtain a map $f^{\prime}: V=\operatorname{Spec} k[t] \rightarrow U$.

Let $P$ be the monoid $\sigma^{\vee} \cap M$, and let $e_{1}, \ldots, e_{l}$ be the irreducible elements of $P$. We see that for each $i$,

$$
f^{*}\left(e_{i}\right)=t^{c_{i}} a_{i},
$$

where $c_{i}$ is the contact order prescribed by $\Gamma$ and $a_{i}$ is some element of $k[t]$. Note that if $\alpha \in k$ is a zero of $a_{i}$, then the point $t=\alpha$ is mapped to the toric boundary; however, the contact order given by $\Gamma$ implies that $t=0$ is the only point in $V$ that maps to the boundary. Hence, $a_{i}$ must be a power of $t$. But if $a_{i}$ is divisible by $t$, then the contact order of $t=0$ along $e_{i}=0$ is greater than $c_{i}$. Therefore, $a_{i}$ must be a nonzero constant.

Now observe that the point $1 \in T \subset U$ is given by $e_{i}=1$ for all $i$. Since $f(1)=1$, the equation $f^{*}\left(e_{i}\right)=t^{c_{i}} a_{i}$ shows that $a_{i}=1$. Note that such $f$ defines a group morphism $\iota_{v}$ as in (1-1). This implies that first statement.

To prove the second statement, it is enough to show that $\mathcal{K}_{\Gamma}^{\circ}(X)$ is irreducible, which again follows from the above statement.

Now we set $\Gamma=\Gamma_{0}$ as in (1-2) and use the setting and notation of the introduction. As discussed in the introduction, we have an immersion $T^{\prime} \rightarrow \mathscr{K}_{\Gamma}(X)$. Let $\mathfrak{X}_{\Gamma}$ be the closure of $T^{\prime}$ in $\mathscr{K}_{\Gamma}(X)$. The forgetful morphism $\Phi$ then induces a map

$$
\phi^{\prime}: \mathfrak{X}_{\Gamma} \rightarrow \mathfrak{M} .
$$

Since $\mathcal{K}_{\Gamma}(X)$ is irreducible, Theorem 1.4 follows from the next lemma.

Lemma 2.4. $\mathfrak{X}_{\Gamma}$ is an open substack of $\mathscr{K}_{\Gamma}(X)$, and so $\phi^{\prime}$ is the normalization map.

Proof. As in the proof of Lemma 2.2, $\phi^{\prime}$ is representable and finite. If $\mathfrak{X}_{\Gamma}$ is an open substack of $\mathscr{K}_{\Gamma}(X)$, it is then normal. Since $\phi^{\prime}$ is an isomorphism over $T^{\prime}$, Zariski's main theorem shows that it is the normalization map.

To show that $\mathfrak{X}_{\Gamma}$ is open in $\mathscr{K}_{\Gamma}(X)$, it suffices to prove that $\mathfrak{X}_{\Gamma}^{\circ}:=\mathfrak{X}_{\Gamma} \cap \mathscr{K}_{\Gamma}^{\circ}(X)$ has the same dimension as $\mathscr{K}_{\Gamma}^{\circ}(X)$. Since $T^{\prime}$ is dense in $\mathfrak{X}_{\Gamma}$, we see that $\mathfrak{X}_{\Gamma}$ has dimension $d-1$. On the other hand, the map

$$
\pi:\left(\mathscr{K}_{\Gamma}(X), M_{\mathscr{K}_{\Gamma}(X)}\right) \rightarrow\left(\mathfrak{M}_{0,2}, M_{\mathfrak{M}_{0,2}}\right)
$$

in the proof of Proposition 2.1 induces a map

$$
\mathcal{K}_{\Gamma}^{\circ}(X) \rightarrow \mathfrak{M}_{0,2}^{\circ},
$$


where $\mathfrak{M}_{0,2}^{\circ}$ denotes the open substack of $\mathfrak{M}_{0,2}$ with smooth fiber curves. By Proposition 2.1, we see that $\mathscr{K}_{\Gamma}^{\circ}(X)$ has dimension $d-1$.

\section{Tropical curves associated to stable log maps}

The goal of this section is to prove Proposition 3.8. Following [Nishinou and Siebert 2006; Gross and Siebert 2013], we explain the connection between tropical curves and stable log maps to toric varieties.

3.1. Review of tropical curves. Let $\bar{G}$ be the geometric realization of a weighted, connected finite graph with weight function $\omega$. That is, $\bar{G}$ is the CW complex associated to a finite connected graph with vertex set $\bar{G}^{[0]}$ and edge set $\bar{G}^{[1]}$, and

$$
\omega: \bar{G}^{[1]} \rightarrow \mathbb{N}
$$

is a function. Here we allow $\bar{G}$ to have divalent vertices. Given an edge $l \in \bar{G}^{[1]}$, we denote its set of adjacent vertices by $\partial l$. If $l$ is a loop, then we require $\omega(l)=0$.

Let $G_{\infty}^{[0]} \subset \bar{G}^{[0]}$ be the set of one-valent vertices, and let

$$
G:=\bar{G} \backslash \bar{G}_{\infty}^{[0]} .
$$

Let $G_{\infty}^{[1]}$ be the set of noncompact edges in $G$, which we refer to as unbounded edges. A flag of $G$ is a pair $(v, l)$ where $l$ is an edge and $v \in \partial l$. We let $F G$ be the set of flags of $G$, and for each vertex $v$, we let

$$
F G(v):=\{(v, l) \in F G\} .
$$

Let $N$ be a lattice and $M=N^{\vee}$. We let $N_{\mathbb{Q}}:=N \otimes_{\mathbb{Z}} \mathbb{Q}$ and $N_{\mathbb{R}}:=N \otimes_{\mathbb{Z}} \mathbb{R}$.

Definition 3.2. A parametrized tropical curve in $N_{\mathbb{Q}}$ is a proper map $\varphi: G \rightarrow N_{\mathbb{R}}$ of topological spaces satisfying the following conditions:

(1) For every edge $l$ of $G$, the restriction $\left.\varphi\right|_{l}$ acts as dilation by a factor $\omega(l)$ with image $\varphi(l)$ contained in an affine line with rational slope. If $\omega(l)=0$, then $\varphi(l)$ is a point.

(2) For every vertex $v$ of $G$, we have $\varphi(v) \in N_{\mathbb{Q}}$.

(3) For each $(v, l) \in F G(v)$, let $u_{v, l}$ be a primitive integral vector emanating from $\varphi(v)$ along the direction of $h(l)$. Then

$$
\epsilon_{v}:=\sum_{(v, l) \in F G(v)} \omega(l) u_{v, l}=0
$$

which we refer to as the balancing condition.

An isomorphism of tropical curves $\varphi: G \rightarrow N_{\mathbb{R}}$ and $\varphi^{\prime}: G^{\prime} \rightarrow N_{\mathbb{R}}$ is a homeomorphism $\Phi: G \rightarrow G^{\prime}$ compatible with the weights of the edges such that $\varphi=\varphi^{\prime} \circ \Phi$.

A tropical curve is an isomorphism class of parametrized tropical curves. 
3.3. Tropical curves from nondegenerate stable log maps. Let $\left(X, \mu_{X}\right)$ be a toric variety with its standard $\log$ structure, and let $T \subset X$ be its defining torus. We denote by $N$ the lattice of one-parameter subgroups of $T$. Let $f:\left(C, \mu_{C}\right) \rightarrow\left(X, \mu_{X}\right)$ be a stable $\log$ map over $\left(S, M_{S}\right)$ with $S$ a geometric point. Further assume that $f$ is nondegenerate; that is, the log structure $M_{S}$ is trivial.

In this subsection, we show how to assign a tropical curve $\operatorname{Trop}(f): G \rightarrow N_{\mathbb{R}}$ to any such nondegenerate stable $\log \operatorname{map} f$. Note that in this case, the points on the source curve with nontrivial log structures are marked points or nodal points. To begin, let $G$ be the graph with a single vertex $v$, which we think of as being associated to the unique component of $C$, and with one unbounded edge for each marked point of $C$. We let $\operatorname{Trop}(f)(v)=0$.

Let $l$ be an edge corresponding to a marked point $p$ of $C$. If $p$ has trivial contact orders, then we set $\omega(l)=0$ and let $\operatorname{Trop}(f)$ contract $l$ to 0 . Otherwise, the contact order is equivalent to giving a nontrivial map

$$
c_{l}: \bar{M}_{X, f(p)} \rightarrow \bar{M}_{C, p}=\mathbb{N} .
$$

Note that we have a surjective cospecialization map of groups

$$
M:=N^{\vee} \rightarrow \bar{M}_{X, f(p)}^{g p}
$$

corresponding to the specialization of the generic point of $T$ to $f(p)$. Composing with $c_{l}^{g p}$, we obtain a map

$$
\mu_{l}: M \rightarrow \mathbb{Z}
$$

which defines an element $\mu_{l} \in N$. Let $u_{l}$ be the primitive vector with slope given by $\mu_{l} \in N$. We define $\omega(l)$ to be the positive integer such that $\mu_{l}=\omega(l) u_{l}$ and define the image $\operatorname{Trop}(f)(l)$ to be the unbounded ray emanating from 0 along the direction of $u_{l}$. This defines our desired map $\operatorname{Trop}(f): G \rightarrow N_{\mathbb{R}}$ up to reparametrization.

Proposition 3.4. $\operatorname{Trop}(f): G \rightarrow N_{\mathbb{R}}$ defines a tropical curve.

Proof. It remains to check that the balancing condition holds. That is, we must show $\epsilon_{v}=0$. Note that every $m \in M$ defines a rational function on $C$ and that the degree of the associated Cartier divisor is $0=\epsilon_{v}(m)$. Therefore, $\epsilon_{v} \in N=M^{\vee}$ is 0 .

\subsection{Tropical curves from stable log maps over the standard log point. Suppose} $\left(X, \mu_{X}\right)$ is a toric variety with its standard log structure, and let $T \subset X$ be its defining torus. Fix discrete data $\Gamma=\left(g, \beta, n,\left\{c_{i}\right\}\right)$, and let $f:\left(C, \mu_{C}\right) \rightarrow\left(X, \mu_{X}\right)$ be a stable log map with discrete data $\Gamma$ over the standard log point $\left(S, M_{S}\right)$; that is, $S$ is a geometric point and $M_{S}$ is the $\log$ structure associated to the map $\mathbb{N} \rightarrow O_{S}$ sending 1 to 0 . This is equivalent to giving a (not necessarily strict) log map

$$
\left(S, M_{S}\right) \rightarrow\left(\mathscr{K}_{\Gamma}(X), M_{\mathscr{K}_{\Gamma}(X)}\right),
$$


and the stable log map $f$ is obtained by pulling back the universal stable log map over $\left(\mathscr{K}_{\Gamma}(X), \mathcal{M}_{\mathscr{K}_{\Gamma}(X)}\right)$. In this subsection, we associate a tropical curve

$$
\operatorname{Trop}(f): G \rightarrow N_{\mathbb{R}}
$$

to $f$ by modifying the construction given in [Gross and Siebert 2013, §1.3].

We define $G$ to be the dual graph of $C$ where we attach an unbounded edge for each marked point. Given a vertex $v$, let $t$ be the generic point of the corresponding component of $C$. We therefore have a morphism

$$
\bar{M}_{X, f(t)} \rightarrow \bar{M}_{C, t}=\mathbb{N}
$$

of monoids. Taking the associated groups and composing with the cospecialization map $M \rightarrow \bar{M}_{X, f(t)}^{g p}$ yields a map

$$
\tau_{v}: M \rightarrow \mathbb{Z}
$$

and hence a point in $N$. We define $\operatorname{Trop}(f)(v)=\tau_{v}$.

Let $l$ be an edge of $G$. If $\partial l=\left\{v, v^{\prime}\right\}$ and $v \neq v^{\prime}$, then we define the image of $l$ under $\operatorname{Trop}(f)$ to be the line segment joining $\tau_{v}$ and $\tau_{v^{\prime}}$. In this case, $\tau_{v^{\prime}}-\tau_{v}=e_{l} \mu_{l}$, where $e_{l} \in \bar{M}_{S}=\mathbb{N}$ is the section that smooths the node corresponding to $l$, and $\mu_{l}$ is an element of $N$. We define $\omega(l)$ to be the positive integer such that $\mu_{l}=\omega(l) u_{l}$, where $u_{l}$ is a primitive integral vector.

Suppose now that $l$ is an unbounded edge corresponding to a marked point $p$. If $p$ has trivial contact orders, then we set $\omega(l)=0$ and let $\operatorname{Trop}(f)$ contract $l$ to $\tau_{v}$, where $\partial l=\{v\}$. Otherwise, the contact orders of $p$ define a nontrivial map

$$
c_{l}: \bar{M}_{X, f(p)} \rightarrow \bar{M}_{C, p}=\mathbb{N} \oplus \bar{M}_{S} \rightarrow \mathbb{N}
$$

where the last map is the projection. Again taking the associated groups and composing with the cospecialization map $M \rightarrow \bar{M}_{X, f(p)}^{g p}$, we obtain

$$
\mu_{l}: M \rightarrow \mathbb{Z} .
$$

We define $\omega(l)$ to be the positive integer such that $\mu_{l}=\omega(l) u_{l}$, where $u_{l} \in N$ is a primitive integral vector, and we let $\operatorname{Trop}(f)(l)$ be the unbounded ray emanating from $\tau_{v}$ in the direction of $u_{l}$.

Proposition 3.6. $\operatorname{Trop}(f): G \rightarrow N_{\mathbb{R}}$ defines a tropical curve.

Proof. We must check that the balancing condition holds for each vertex $v$ of $G$. As in the proof of Proposition 3.4, every $m \in M$ defines a rational function on the irreducible component of $C$ corresponding to $v$. The associated Cartier divisor has degree $0=\epsilon_{v}(m)$, and so $\epsilon_{v}=0$; see [Gross and Siebert 2013, Proposition 1.14]. 
Remark 3.7. Let $R$ be the complete local ring of $\mathbb{A}^{1}$ at the origin, and let $\mu_{R}$ be the $\log$ structure on $R$ induced by the standard $\log$ structure on $\mathbb{A}^{1}$. Denote the closed and generic points of Spec $R$ by 0 and $\eta$, respectively. Suppose $h:\left(\mathscr{C}, \mathcal{M}_{\mathscr{C}}\right) \rightarrow\left(X, \mathcal{M}_{X}\right)$ is a stable log map over $R$ with discrete data $\Gamma$ such that $h_{0}=f$. Note that $h_{\eta}$ is a nondegenerate stable log map. For each marked section $p$ : Spec $R \rightarrow \mathscr{C}$, let $l_{0}$ and $l_{\eta}$ be the edges of the dual graphs of $\mathscr{C}_{0}$ and $\mathscr{C}_{\eta}$ corresponding to the marked points $p_{0}$ and $p_{\eta}$, respectively. Consider the morphism

$$
\left.\left.\bar{M}_{X}\right|_{h(p)} \rightarrow \bar{M}_{\mathscr{C}}\right|_{p}=\mathbb{N} \oplus \bar{M}_{R} \rightarrow \mathbb{N},
$$

where the last map is the projection. Taking associated groups and precomposing with the map $\left.M \rightarrow \bar{M}_{X}^{g p}\right|_{h(p)}$, we obtain a map $M \rightarrow \mathbb{Z}$ of constant sheaves on Spec $R$ whose special and generic fibers are $\mu_{l_{0}}$ and $\mu_{l_{\eta}}$. Hence, we see $\mu_{l_{0}}=\mu_{l_{\eta}}$.

Denote by $\operatorname{Trop}\left(f_{\eta}\right)$ and $\operatorname{Trop}\left(f_{0}\right)$ the tropical curves associated to the generic and closed fiber of $f$. Then the above argument implies that $\operatorname{Trop}\left(f_{\eta}\right)$ is the asymptotic fan of $\operatorname{Trop}\left(f_{0}\right)$ as defined in [Nishinou and Siebert 2006, Definition 3.1].

The following result plays an important role in the proof of Theorem 1.1:

Proposition 3.8. If the discrete data $\Gamma$ is given by $g=0, n=2$, and $\beta \neq 0$, then $\operatorname{Trop}(f)$ is an embedding whose image is a line. Moreover, $C$ is a chain of $\mathbb{P}^{1} s$ and $f$ does not contract any components of $C$.

Proof. Since $\mathscr{K}_{\Gamma}(X)$ is log smooth by Proposition 2.1, there exists a stable log map $h:\left(\mathscr{C}, M_{\mathscr{C}}\right) \rightarrow\left(X, \mu_{X}\right)$ over $\left(R, \mu_{R}\right)$ as in Remark 3.7. Let $p, p^{\prime}: \operatorname{Spec} R \rightarrow \mathscr{C}$ be the two marked sections, and let $l_{0}, l_{0}^{\prime}, l_{\eta}$, and $l_{\eta}^{\prime}$ be the corresponding edges of the dual graphs of $C$ and $\mathscr{C}_{\eta}$. Since $\beta \neq 0$, the two marked points $p_{\eta}$ and $p_{\eta}^{\prime}$ of $\mathscr{b}_{\eta}$ have nontrivial contact orders. The balancing condition for $\operatorname{Trop}\left(h_{\eta}\right)$ then shows $\mu_{l_{\eta}^{\prime}}=-\mu_{l_{\eta}} \neq 0$. By Remark 3.7, we therefore have $\mu_{l_{0}^{\prime}}=-\mu_{l_{0}} \neq 0$. In particular, $\operatorname{Trop}(f)$ maps $l_{0}$ and $l_{0}^{\prime}$ to unbounded rays.

We next show that if $l$ is an edge of $G$, then $\operatorname{Trop}(f)(l)$ is a point or it is a line segment or ray parallel to $\mu_{l_{0}}$. Suppose $\operatorname{Trop}(f)(l)$ is not a point. If $\operatorname{Trop}(f)(l)$ is unbounded, then $l$ is $l_{0}$ or $l_{0}^{\prime}$, and so $\operatorname{Trop}(f)(l)$ is parallel to $\mu_{l_{0}}$. Otherwise, $\operatorname{Trop}(f)(l)$ is a line segment and $\partial l=\left\{v, v_{1}\right\}$ with $v \neq v_{1}$. If $\operatorname{Trop}(f)(l)$ is not parallel to $\mu_{l_{0}}$, then the balancing condition shows that there is an edge $l_{1} \neq l$ such that $v_{1} \in \partial l_{1}$ and $\operatorname{Trop}(f)\left(l_{1}\right)$ is not parallel to $\mu_{l_{0}}$. Hence, $l_{1}$ is a line segment with endpoints $v_{1}$ and $v_{2}$. Again, the balancing condition shows that there is an edge $l_{2}$ containing $v_{2}$ such that $\operatorname{Trop}(f)\left(l_{2}\right)$ is a line segment which is not parallel to $\mu_{l_{0}}$. Since $C$ has genus 0 , we see $l, l_{1}$, and $l_{2}$ are distinct. Continuing in this manner, we produce an infinite sequence of distinct edges $l_{i}$ of the dual graph of $C$. This is a contradiction.

Lastly, we show that every irreducible component $A$ of $C$ has exactly two special points. Hence, $C$ is a chain of $\mathbb{P}^{1} \mathrm{~s}, f$ does not contract any component of $C$, 
and $\operatorname{Trop}(f)(G)$ is a line parallel to $\mu_{l_{0}}$. Suppose $A$ is a component with at least three special points, and let $v$ be the vertex of $G$ corresponding to $A$. Then $G \backslash v$ is a disjoint union of nonempty trees $T_{1}, T_{2}, \ldots, T_{m}$ with $m \geq 3$. Without loss of generality, $T_{1}$ only contains bounded edges. The argument in the preceding paragraph then shows that $\operatorname{Trop}(f)$ maps every edge of $T_{1}$ to a single point. If $C_{1}$ denotes the subcurve of $C$ corresponding to $T_{1}$, then we see that every special point of $C_{1}$ has a trivial contact order, and so $f$ contracts $C_{1}$. Since $T_{1}$ is a tree, $C_{1}$ contains components with only two special points. This contradicts the stability of $f$.

\section{The Chow quotient as the coarse moduli space}

Throughout this section, we let $\Gamma=\Gamma_{0}$ and $C(X)$ denote the Chow variety as in the introduction. Let $K$ be the normalization of $X / / T_{0}$. Since the stack $\mathscr{K}_{\Gamma}(X)$ is normal, it follows from [Kollár 1996, Chapter I, 3.17 and 3.21] that there is a map

$$
F: \mathscr{K}_{\Gamma}(X) \rightarrow C(X)
$$

sending a stable $\log$ map $f:\left(C, \mu_{C}\right) \rightarrow\left(X, \mu_{X}\right)$ to the image cycle $f_{*}[C]$. Since $\mathscr{K}_{\Gamma}(X)$ is irreducible by Theorem $1.4, F$ factors as

$$
\mathcal{K}_{\Gamma}(X) \stackrel{F^{\prime}}{\rightarrow} X / / T_{0} \stackrel{i}{\rightarrow} C(X),
$$

where $i$ is the natural inclusion. Since $F$ is an isomorphism over $T^{\prime}$ and $\mathscr{K}_{\Gamma}(X)$ is normal, by Proposition 2.1, we obtain an induced morphism

$$
G: \mathscr{K}_{\Gamma}(X) \rightarrow K
$$

To prove Theorem 1.1, we show:

Proposition 4.1. $G$ is a coarse space morphism.

Proof. Note that both $\mathscr{K}_{\Gamma}(X)$ and $K$ are normal and proper, and $G$ is bijective on the level of closed points over $T^{\prime}$. To show that $K$ is the coarse moduli space of $\mathscr{K}_{\Gamma}(X)$, by Zariski's main theorem, it suffices to show $G$ is quasifinite. To do so, it is enough to show $F^{\prime}$ is quasifinite at the level of closed points. That is, we show that if $x \in X / / T_{0}$ is a closed point and $E_{x}$ denotes the corresponding cycle of $X$, then there are finitely many stable log maps whose image cycles are given by $E_{x}$. Let

$$
E_{x}=\sum a_{i} Z_{i}
$$

where the $a_{i}$ are positive integers and the $Z_{i}$ are reduced irreducible closed subschemes of $X$. Let $\widetilde{Z}_{i}$ be the normalization of $Z_{i}$. Since $E_{x}$ is of dimension 1 , we have $\widetilde{Z}_{i} \simeq \mathbb{P}^{1}$.

We claim that if $f:\left(C, \mu_{C}\right) \rightarrow\left(X, M_{X}\right)$ is a stable log map that defines a closed point of $\mathscr{K}_{\Gamma}(X)$ such that the image cycle of $f$ is $E_{x}$, then $f$ can only be 
ramified at the special points of $C$. Given this claim, $F^{\prime}$ is quasifinite. Indeed, since Proposition 3.8 shows that no component of $C$ is contracted under $f$, the number of irreducible components of $C$ is bounded by $\sum a_{i}$. For each irreducible component $A$ of $C$, the restriction $\left.f\right|_{A}$ factors as

$$
A \rightarrow \widetilde{Z}_{i} \rightarrow X
$$

for some $i$. Since the first map $A \rightarrow \widetilde{Z}_{i}$ can only be ramified at the two fixed special points, it is determined by the degree of $\left.f\right|_{A}$. This implies that there are only finitely many choices for the underlying map $C \rightarrow X$. Since the forgetful morphism $\Phi: \mathscr{K}_{\Gamma}(X) \rightarrow \mathfrak{M}_{0,2}(X, \beta)$ is finite, there are finitely many choices for the stable $\log$ map $f$.

It remains to prove the claim. By Proposition $2.3, \mathscr{K}_{\Gamma}(X)$ is irreducible and $T^{\prime}$ is dense, so there exists a toric morphism $\mathbb{A}^{1} \rightarrow \mathscr{K}_{\Gamma}(X)$ such that the fiber over $0 \in \mathbb{A}^{1}$ is our given stable log map $f:\left(C, \mu_{C}\right) \rightarrow\left(X, \mu_{X}\right)$ whose image cycle is $E_{x}$. Let $R$ denote the complete local ring $\widehat{\widehat{O}}_{\mathbb{A}^{1}, 0}$ and let

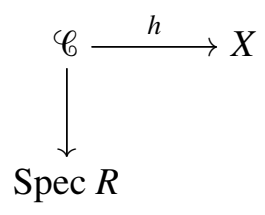

be the associated underlying stable map. Let $\eta \in \operatorname{Spec} R$ be the generic point.

We first handle the case when $X$ is smooth. Let $\mathscr{C}^{\circ}$ be the open subset of $\mathscr{C}$ obtained by removing the special points. Note that $\mathscr{C}^{\circ}$ is normal, and $\left.h\right|_{\mathscr{C}^{\circ}}$ is quasifinite by Proposition 3.8. By the purity of the branch locus theorem [Altman and Kleiman 1971, p. 461], if $\left.h\right|_{\mathscr{C}^{\circ}}$ is ramified, then the ramification locus $D$ is pure of codimension 1 . Since $\left.h\right|_{\mathscr{C}^{\circ}}$ is not everywhere ramified over the central fiber, $D$ must intersect the generic fiber. However, $\left.h\right|_{\mathscr{C}^{\circ}}$ is unramified over the generic fiber, so we conclude that $D$ is empty.

We now consider the case when $X$ is singular. Let $p: \widetilde{X} \rightarrow X$ be a toric resolution. We may replace $R$ by a ramified extension as this does not affect the set of closed points. By the properness of $\mathscr{K}_{\Gamma}(X)$, we can assume we have a stable log map $\tilde{h}:\left(\widetilde{\mathscr{C}}, M_{\widetilde{\mathscr{C}}}\right) \rightarrow\left(\widetilde{X}, M_{\tilde{X}}\right)$ and a commutative diagram of the underlying maps

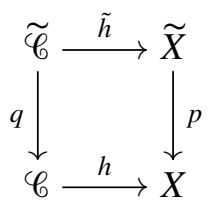

over $R$. Here $h$ is the underlying map of the stable log map to $X$, which can be also obtained by taking the stabilization of the prestable map $p \circ \tilde{h}$. The previous 
paragraph shows that $\tilde{h}$ only ramifies at the special points. Since Proposition 3.8 shows that $\widetilde{\mathscr{C}}$ and $\mathscr{C}$ are both chains of $\mathbb{P}^{1}$ s, we see that $h$ only ramifies at the special points as well.

\section{Appendix: Toric varieties have generalized Deligne-Faltings log structures}

The theory of moduli spaces of stable log maps $\mathscr{K}_{\Gamma}\left(Y, M_{Y}\right)$ is developed in [Chen 2011; Abramovich and Chen 2011] and [Gross and Siebert 2013] for different classes of $\log$ schemes $\left(Y, M_{Y}\right)$. In [Chen 2011; Abramovich and Chen 2011], Abramovich and the first author consider log schemes that are generalized DeligneFaltings (see Definition A.1); Gross and Siebert [2013] consider log schemes that are quasigenerated Zariski. It is shown in [Abramovich and Chen 2011, Proposition 4.8] that when $\left(Y, M_{Y}\right)$ is both generalized Deligne-Faltings and quasigenerated Zariski, the Abramovich-Chen and Gross-Siebert constructions are identical. Gross and Siebert show that the standard $\log$ structure $M_{X}$ on a normal toric variety $X$ is always quasigenerated Zariski. Here we show that if $X$ is also projective, then $\mu_{X}$ is generalized Deligne-Faltings. Therefore, the two theories agree for projective normal toric varieties.

Definition A.1. A log structure $M_{Y}$ on a scheme $Y$ is called generalized DeligneFaltings if there exists a fine saturated sharp monoid $P$ and a morphism $P \rightarrow \bar{M}_{Y}$ that locally lifts to a chart $P \rightarrow M_{Y}$.

Remark A.2. Given a fine saturated sharp monoid $P$, let $A_{P}=\operatorname{Spec} k[P]$ with its standard $\log$ structure $M_{A_{P}}$. Then there is a natural action of $T_{P}:=\operatorname{Spec} k\left[P^{g p}\right]$ on $\left(A_{P}, M_{A_{P}}\right)$ induced by the morphism $P \rightarrow P \oplus P^{g p}$ sending $p$ to $(p, p)$. The $\log$ structure $M_{A_{P}}$ descends to yield a log structure $\mathcal{M}_{\left[A_{P} / T_{P}\right]}$ on the quotient stack $\left[A_{P} / T_{P}\right]$. By [Olsson 2003, Remark 5.15], a log scheme $\left(Y, M_{Y}\right)$ is generalized Deligne-Faltings if and only if there exists a strict morphism

$$
\left(Y, M_{Y}\right) \rightarrow\left(\left[A_{P} / T_{P}\right], M_{\left[A_{P} / T_{P}\right]}\right)
$$

for some fine saturated sharp monoid $P$.

Let $X$ be a projective normal toric variety, and let $\mu_{X}$ be its standard log structure. Let $Q \subset \mathbb{R}^{n}$ be a polytope associated to a sufficiently positive projective embedding of $X$. Placing $Q$ at height 1 in $\mathbb{R}^{n} \times \mathbb{R}$ and letting $P$ be the monoid of lattice points in the cone over $Q$, we have $X=\operatorname{Proj} k[P]$. Note that $P$ is fine, saturated, and sharp. Let $\left(A_{P}, M_{A_{P}}\right)$ be as in Remark A.2, let $U$ be the compliment of the closed subscheme of $A_{P}$ defined by the irrelevant ideal of $k[P]$, and let $M_{U}=\left.M_{A_{P}}\right|_{U}$. The function deg $: P \rightarrow \mathbb{Z}$ sending an element to its height induces a $\mathbb{G}_{m}$-action on $\left(A_{P}, M_{A_{P}}\right)$. Hence, $M_{U}$ descends to yield a log structure $M_{P}$ on $X$.

Lemma A.3. $\mu_{P}$ is generalized Deligne-Faltings. 
Proof. We have a cartesian diagram

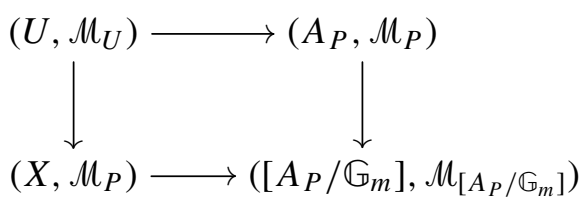

where all morphisms are strict and the vertical morphisms are smooth covers. Note that the $\mathbb{G}_{m}$-action on $\left(A_{P}, M_{A_{P}}\right)$ is induced from the morphism $\sigma: P \rightarrow P \oplus \mathbb{Z}$ defined by $p \mapsto(p, \operatorname{deg} p)$. Since $\sigma$ factors as

$$
P \rightarrow P \oplus P^{g p} \rightarrow P \oplus \mathbb{Z}
$$

where the first map is $p \mapsto(p, p)$ and the second is $(p, \xi) \mapsto(p, \operatorname{deg} \xi)$, we see that there is a strict smooth cover

$$
\left(\left[A_{P} / \mathbb{G}_{m}\right], M_{\left[A_{P} / \mathbb{G}_{m}\right]}\right) \rightarrow\left(\left[A_{P} / T_{P}\right], M_{\left[A_{P} / T_{P}\right]}\right) .
$$

Hence, Remark A.2 shows that $\mu_{P}$ is generalized Deligne-Faltings.

Note that $\left.M_{P}\right|_{T}=O_{T}^{*}$, where $T$ is the torus of $X$. We therefore obtain a map

$$
\psi: \mathcal{M}_{P} \rightarrow j_{*}^{\log } \mathrm{O}_{T}^{*}=: \mu_{X}
$$

Proposition A.4. $\psi$ is an isomorphism, and so $\left(X, \mu_{X}\right)$ is generalized DeligneFaltings.

Proof. To show $\psi$ is an isomorphism, it is enough to look Zariski locally on $X$. Note that $X$ has an open cover by the $X_{v}:=\operatorname{Spec} k\left[Q_{v}\right]$, where $v$ is a vertex of the polytope $Q$ and $Q_{v}$ is the monoid of lattice points in the cone over $Q-v:=$ $\left\{q-v \mid q \in Q \subset \mathbb{R}^{n}\right\}$. Let $P_{v}$ be the submonoid of $P^{g p}$ generated by $P$ and $-v$. Then we have a cartesian diagram

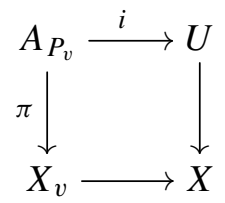

where $\pi$ is induced from the map $Q_{v} \rightarrow P_{v}$ embedding $Q_{v}$ at height 0 in $P_{v}$ and where the composite of $i$ and $U \rightarrow A_{P}$ is induced from the inclusion $P \rightarrow P_{v}$. Hence,

$$
M_{Q_{v}}=\left(M_{P_{v}}\right)^{\mathbb{G}_{m}}
$$

and so $\psi$ is an isomorphism over $X_{v}$. 


\section{Acknowledgments}

We would like to thank Dan Abramovich, Dustin Cartwright, Anton Geraschenko, Noah Giansiracusa, and Martin Olsson. We also thank the anonymous referee for helpful comments. Chen was partially supported by the Simons Foundation. Satriano was partially supported by NSF grant DMS-0943832 and an NSF postdoctoral fellowship (DMS-1103788).

\section{References}

[Abramovich and Chen 2011] D. Abramovich and Q. Chen, "Stable logarithmic maps to DeligneFaltings pairs, II", preprint, 2011. arXiv 1102.4531v2

[Abramovich et al. 2011] D. Abramovich, Q. Chen, W. D. Gillam, and S. Marcus, "The evaluation space of logarithmic stable maps", preprint, 2011. arXiv 1012.5416v1

[Abramovich et al. $\geq 2013$ ] D. Abramovich, Q. Chen, M. Gross, and B. Siebert, in preparation.

[Altman and Kleiman 1971] A. Altman and S. L. Kleiman, "On the purity of the branch locus", Compos. Math. 23 (1971), 461-465. MR 46 \#7233 Zbl 0242.14001

[Chen 2011] Q. Chen, "Stable logarithmic maps to Deligne-Faltings pairs, I", preprint, 2011. arXiv 1008.3090v4

[Gross and Siebert 2013] M. Gross and B. Siebert, "Logarithmic Gromov-Witten invariants", J. Amer. Math. Soc. 26:2 (2013), 451-510. MR 3011419 Zbl 06168513

[Kapranov et al. 1991] M. M. Kapranov, B. Sturmfels, and A. V. Zelevinsky, "Quotients of toric varieties”, Math. Ann. 290:4 (1991), 643-655. MR 92g:14050 Zbl 0762.14023

[Kato 1989] K. Kato, "Logarithmic structures of Fontaine-Illusie", pp. 191-224 in Algebraic analysis, geometry, and number theory (Baltimore, 1988), edited by J.-I. Igusa, Johns Hopkins Univ. Press, Baltimore, MD, 1989. MR 99b:14020 Zbl 0776.14004

[Kato 1996] F. Kato, "Log smooth deformation theory", Tohoku Math. J. (2) 48:3 (1996), 317-354. MR 99a:14012 Zbl 0876.14007

[Kato 2000] F. Kato, "Log smooth deformation and moduli of log smooth curves", Internat. J. Math. 11:2 (2000), 215-232. MR 2001d:14016 Zbl 1100.14502

[Kollár 1996] J. Kollár, Rational curves on algebraic varieties, Ergebnisse der Mathematik und ihrer Grenzgebiete (3) 32, Springer, Berlin, 1996. MR 98c:14001 Zbl 00833161

[Nishinou and Siebert 2006] T. Nishinou and B. Siebert, "Toric degenerations of toric varieties and tropical curves”, Duke Math. J. 135:1 (2006), 1-51. MR 2007h:14083 Zbl 1105.14073

[Nizioł 2006] W. Nizioł, "Toric singularities: log-blow-ups and global resolutions", J. Algebraic Geom. 15:1 (2006), 1-29. MR 2006i:14015 Zbl 1100.14011

[Ogus 2006] A. Ogus, "Lectures on logarithmic algebraic geometry", preprint, 2006, available at http://math.berkeley.edu/ ogus/preprints/log_book/logbook.pdf.

[Olsson 2003] M. C. Olsson, "Logarithmic geometry and algebraic stacks", Ann. Sci. École Norm. Sup. (4) 36:5 (2003), 747-791. MR 2004k:14018 Zbl 1069.14022

[Olsson 2007] M. C. Olsson, “(Log) twisted curves”, Compos. Math. 143:2 (2007), 476-494. MR 2008d:14021 Zbl 1138.14017

[Olsson 2008] M. Olsson, "Logarithmic interpretation of the main component in toric Hilbert schemes", pp. 231-252 in Curves and abelian varieties, edited by V. Alexeev et al., Contemp. Math. 465, Amer. Math. Soc., Providence, RI, 2008. MR 2009j:14007 Zbl 1153.14007 
[Peeva and Stillman 2002] I. Peeva and M. Stillman, "Toric Hilbert schemes", Duke Math. J. 111:3 (2002), 419-449. MR 2003m:14008 Zbl 1067.14005

Communicated by Ravi Vakil

Received 2012-10-22 Revised 2013-02-04 Accepted 2013-03-12

q-chen@math.columbia.edu Department of Mathematics, Columbia University, 2990 Broadway, New York, NY 10027, United States

satriano@umich.edu Department of Mathematics, University of Michigan, 2074 East Hall, Ann Arbor, MI 48109, United States 


\section{Algebra \& Number Theory}

msp.org/ant

\section{EDITORS}

MANAGING EDITOR

Bjorn Poonen

Massachusetts Institute of Technology

Cambridge, USA

\author{
EDITORIAL BOARD CHAIR \\ David Eisenbud \\ University of California \\ Berkeley, USA
}

\section{BOARD OF EDITORS}

Georgia Benkart

Dave Benson

Richard E. Borcherds

John H. Coates

J-L. Colliot-Thélène

Brian D. Conrad

Hélène Esnault

Hubert Flenner

Edward Frenkel

Andrew Granville

Joseph Gubeladze

Roger Heath-Brown

Ehud Hrushovski

Craig Huneke

Mikhail Kapranov

Yujiro Kawamata

János Kollár

Yuri Manin

Barry Mazur

Philippe Michel
University of Wisconsin, Madison, USA

University of Aberdeen, Scotland

University of California, Berkeley, USA

University of Cambridge, UK

CNRS, Université Paris-Sud, France

University of Michigan, USA

Freie Universität Berlin, Germany

Ruhr-Universität, Germany

University of California, Berkeley, USA

Université de Montréal, Canada

San Francisco State University, USA

Oxford University, UK

Hebrew University, Israel

University of Virginia, USA

Yale University, USA

University of Tokyo, Japan

Princeton University, USA

Northwestern University, USA

Harvard University, USA

École Polytechnique Fédérale de Lausanne
Susan Montgomery

Shigefumi Mori

Raman Parimala

Jonathan Pila

Victor Reiner

Karl Rubin

Peter Sarnak

Joseph H. Silverman

Michael Singer

Vasudevan Srinivas

J. Toby Stafford

Bernd Sturmfels

Richard Taylor

Ravi Vakil

Michel van den Bergh

Marie-France Vignéras

Kei-Ichi Watanabe

Efim Zelmanov

Shou-Wu Zhang
University of Southern California, USA

RIMS, Kyoto University, Japan

Emory University, USA

University of Oxford, UK

University of Minnesota, USA

University of California, Irvine, USA

Princeton University, USA

Brown University, USA

North Carolina State University, USA

Tata Inst. of Fund. Research, India

University of Michigan, USA

University of California, Berkeley, USA

Harvard University, USA

Stanford University, USA

Hasselt University, Belgium

Université Paris VII, France

Nihon University, Japan

University of California, San Diego, USA

Princeton University, USA

PRODUCTION

production@msp.org

Silvio Levy, Scientific Editor

See inside back cover or msp.org/ant for submission instructions.

The subscription price for 2013 is US $\$ 200 /$ year for the electronic version, and $\$ 350 /$ year $(+\$ 40$, if shipping outside the US) for print and electronic. Subscriptions, requests for back issues and changes of subscribers address should be sent to MSP.

Algebra \& Number Theory (ISSN 1944-7833 electronic, 1937-0652 printed) at Mathematical Sciences Publishers, 798 Evans Hall \#3840, c/o University of California, Berkeley, CA 94720-3840 is published continuously online. Periodical rate postage paid at Berkeley, CA 94704, and additional mailing offices.

ANT peer review and production are managed by EditFLOW ${ }^{\circledR}$ from Mathematical Sciences Publishers.

\section{PUBLISHED BY}

- mathematical sciences publishers

nonprofit scientific publishing

http://msp.org/

(C) 2013 Mathematical Sciences Publishers 


\section{Algebra \& Number Theory}

Volume $7 \quad$ No. $9 \quad 2013$

Multiplicities associated to graded families of ideals Steven Dale Cutkosky

Normal coverings of linear groups

JOHN R. BRITNELL and ATTILA MARÓTI

Modularity of the concave composition generating function

George E. ANDrews, Robert C. RhOAdes and SANDER P. ZWEgERS

ANDREW NILES

Regular permutation groups of order $m p$ and Hopf Galois structures

2203

ТIMOTHY KOHL

Further evidence for conjectures in block theory

2241

BENJAMIN SAMBALE

Network parametrizations for the Grassmannian

2275

Kelli TALASKa and LAUREN Williams

Chow quotients of toric varieties as moduli of stable log maps

2313

Qile Chen and Matthew Satriano

Vinberg's representations and arithmetic invariant theory

JACK A. THORNE 